\title{
Successive Linearisation Solution of Free Convection Non-Darcy Flow with Heat and Mass Transfer
}

\author{
Sandile Motsa ${ }^{1}$ and Stanford Shateyi ${ }^{2}$ \\ ${ }^{1}$ University of Swaziland \\ ${ }^{2}$ University of Venda \\ ${ }^{1}$ Swaziland \\ ${ }^{2}$ South Africa
}

\section{Introduction}

Coupled convective heat and mass transfer processes in porous media has received a great deal of attention amongst researchers in recent years because of its numerous applications. The heat and mass tranfer phenomena in porous media is often encountered in industrial and engineering applications including transport processes through packed bed reactors in the chemical industry, ground water flows, oil industry, nuclear reactors, ceramic industry and in many other applications where heat and mass transfer with convection in porous media can be observed. Examples of recent studies where heat and mass transfer in porous media has been analysed include Amin (2004); Murthty \& Singh (1999); Narayana \& Murthy (2006); Partha (2008).

The boundary layer equations governing the non-Darcy flow with heat and mass transfer are highly non-linear and do not usually possess exact analytical solutions. In recent years, a great deal of attention has been focussed towards developing new methods of finding solutions to non-linear boundary value problems (BVPs) in general. Traditional numerical techniques for solving non-linear BVPs arising in fluid mechanics and other engineering applications include finite differences, finite element methods, Keller-Box method, Runge-Kutta with shooting methods, and spectral collocation methods. Recent advances in computational fluid dynamics and the advent of inexpensive high-speed computers have resulted in the creation of powerful easy-to-use software tools which make use of some of the traditional numerical techniques. Solving complex non-linear BVPs can now be done in a matter of seconds using functions or routines which are built into scientific computation software packages such as Maple, Matlab, Mathematica, Scilab and many others. Despite their apparent simplicity and power, these in-built functions along with the numerical methods have their own limitations such as dealing with problems with singularities, problems with multiple solutions, problems requiring stringent accuracies and for problems with solutions that have very sharp changes. Besides, from numerical results, it is also difficult to have a clear understanding and give essential interpretation of the numerically generated solutions of the underlying non-linear problem. 
Apart from the traditional numerical methods for solving boundary layer equations recent purely analytic or semi-analytic approaches have been developed. These include the Adomian decomposition method Adomian (1976; 1991), homotopy perturbation method He (1999; 2000; 2006), homotopy analysis method Liao (1992; 1999; 2003; 2009), differential transform methods Zhou (1986); Chen \& Ho (1999); Ayaz (2004), variational iteration method He (1999) . Apart from the homotopy analysis method (HAM), these methods converge very slowly or fail to converge at all in highly non-linear problems with very large parameters. The advantage of the HAM is that it uses a convergence controlling parameter which may accelerate convergence to the solution of the non-linear problem. But, in some cases, even with the use of this convergence controlling parameter, convergence of the HAM may be very slow in problems with very large parameters and in problems defined on unbounded domains such as the boundary layer problems. The mathematical difficulties associated with non-linear boundary layer equations have prompted several researchers to continually seek ways of improving on these numerical and analytic solution methods. A strategy that is commonly used to address the problems of slow convergence of analytical solutions is to use the Padé technique such as in the Hermite Padé and Homotopy Padé approach. A novel idea of blending spectral collocation methods and the homotopy analysis method to obtain the spectral-homotopy analysis method (SHAM) has recently been proposed in Motsa et al. $(2010 a ; b)$. The advantage of the SHAM is that it uses the best ideas from both the analytic and numerical approaches such as the use of convergence controlling parameters (from the HAM) and the promise of spectral accuracy and the ability to deal with coupled equations (from the spectral collocation methods).

The aim of this work is to present a new numerical perturbation scheme for solving complex nonlinear boundary value problems arising in problems of heat and mass transfer. The proposed method of solution, hereinafter referred to as the successive linearisation method (SLM), is based on a novel idea of iteratively linearising the underlying governing non-linear boundary layer equations, which are written in similarity form, and solving the resulting equations using spectral methods. The SLM approach has been successfully applied to different fluid flow problems (see for example Motsa et al. (2010c); Makukula et al. (2010a;b); Shateyi \& Motsa (2010)). The flow velocity, temperature and concentration profiles, skin friction and rate of surface heat and transfer are computed using the proposed method. The influence of the governing parameters on these flow characteristics is illustrated graphically and using tables. The results of the proposed method are validated by comparing them with some available results published in literature and against results obtained using other numerical solutions. The comparison indicates that the proposed SLM approximation converges very rapidly to the true solutions and is at least as good as or more accurate than the most efficient methods of solutions currently being used. Because of its simplicity, rapid convergence and accuracy, we conclude that the proposed method of successive linearisation has great potential of being used in other related studies in non-linear science and engineering. The SLM method can therefore be used in place of traditional methods such as finite differences, Runge-Kutta shooting methods, finite elements, etc, in solving non-linear boundary value problems.

\section{Mathematical formulation}

We consider the problem of free convection heat and mass transfer from a vertical surface embedded in a fluid saturated non-Darcy porous medium. The $x$ - axis is along the wall surface and the $y$ - axis is normal to it. The wall is maintained at a constant temperature $T_{w}$ 
and concentration $C_{w}$ which are greater than the ambient temperature $T_{\infty}$ and concentration $C_{\infty}$, respectively. The governing equations for the steady non-Darcy flow can be written as

$$
\begin{aligned}
\frac{\partial u}{\partial x}+\frac{\partial v}{\partial y} & =0 \\
\frac{\partial u}{\partial y}+\frac{c \sqrt{K}}{v} \frac{\partial\left(u^{2}\right)}{\partial y} & =\frac{K g}{v}\left[\beta_{T} \frac{\partial T}{\partial y}+\beta_{C} \frac{\partial C}{\partial y}\right] \\
u \frac{\partial T}{\partial x}+v \frac{\partial T}{\partial y} & =\alpha \frac{\partial^{2} T}{\partial y^{2}}+\frac{D_{m} K_{T}}{c_{s} c_{p}} \frac{\partial^{2} C}{\partial y^{2}} \\
u \frac{\partial C}{\partial x}+v \frac{\partial C}{\partial y} & =D_{m} \frac{\partial^{2} C}{\partial y^{2}}+\frac{D_{m} K_{T}}{T_{m}} \frac{\partial^{2} T}{\partial y^{2}}
\end{aligned}
$$

The boundary conditions are defined as follows;

$$
\begin{gathered}
v=0, \quad T=T_{w}, C=C_{w}, \quad \text { at } y=0, \\
u=0, \quad T=T_{\infty}, C=C_{\infty}, \quad \text { as } y \Rightarrow \infty,
\end{gathered}
$$

where $u$ and $v$ denote velocity components in the $x$ - and $y$ - directions, respectively, $T$ and $C$ are the temperature and concentration, respectively, $K$ is the permeability parameter, $\beta_{T}$ and $\beta_{C}$ are the coefficients of thermal and solutal expansions, respectively, $c$ is the Forchheimer constant, $v$ is the kinematic viscosity, $g$ is the acceleration due to gravity, $\alpha$ and $D_{m}$ are the thermal and mass diffusivity, respectively, $c_{p}$ is the specific heat capacity, $c_{s}$ is the concentration susceptibility, $T_{m}$ is the mean fluid temperature and $K_{T}$ is the thermal diffusion ratio.

By making use of the following similarity variable transformations,

$$
\begin{aligned}
\eta & =\frac{y}{x} \sqrt{R a_{x}}, u=\frac{\alpha}{x} R a_{x} f^{\prime}(\eta), v=-\frac{\alpha}{2 x} \sqrt{R a_{x}}\left[f(\eta)-\eta f^{\prime}(\eta)\right], \\
\theta(\eta) & =\frac{T-T_{\infty}}{T_{w}-T_{\infty}}, \phi(\eta)=\frac{C-C_{\infty}}{C_{w}-C_{\infty}},
\end{aligned}
$$

the governing equations $(1-6)$ are reduced to the following system of non-linear ordinary differential equations,

$$
\begin{aligned}
f^{\prime \prime}+2 G r^{*} f^{\prime} f^{\prime \prime} & =\theta^{\prime}+N_{1} \phi^{\prime}, \\
\theta^{\prime \prime}+\frac{1}{2} f \theta^{\prime}+D_{f} \phi^{\prime \prime} & =0, \\
\phi^{\prime \prime}+\frac{1}{2} \operatorname{Lef}^{\prime}+\operatorname{LeS}_{r} \theta^{\prime \prime} & =0 .
\end{aligned}
$$

The transformed dimensionless boundary conditions are:

$$
\begin{gathered}
f=0, \quad \theta=1, \quad \phi=1, \text { at } \eta=0 \\
f^{\prime}=0, \quad \theta=0, \quad \phi=0, \text { as } \eta \rightarrow \infty
\end{gathered}
$$


where the primes denote differentiation with respect to $\eta$. In equations $(9-11)$

$G r^{*}=c \sqrt{K} K g \beta_{T}\left(T_{w}-T_{\infty}\right) / v^{2}$ represents the inertial parameter, $R a_{x}=K g \beta_{T}\left(T_{w}-T_{\infty}\right) x / \alpha v$ is the Darcy-Rayleigh number, $N_{1}=\frac{\beta_{c}\left(C_{w}-C_{\infty}\right)}{\beta_{T}\left(T_{w}-T_{\infty}\right)}$ is the buoyancy ratio, $L e=\alpha / D_{m}$ is the Lewis number, $D_{f}=\frac{D m K_{T}\left(C_{w}-C_{\infty}\right)}{\alpha c_{s} c_{p}}$ is the Dufour number and $S_{r}=\frac{K_{T} D_{m}\left(T_{w}-T_{\infty}\right)}{\alpha T_{m}\left(C_{w}-C_{\infty}\right)}$ is the Soret number. Other parameters of engineering interest for the present problem are the local Nusselt number and the local Sherwood number, which are given in dimensionless terms by the expressions

$$
N u_{x} / R a_{x}^{1 / 2}=-\theta^{\prime}(0), \quad S h_{x} / R a_{x}^{1 / 2}=-\phi^{\prime}(0)
$$

Equations (9 - 11) are fully coupled and highly nonlinear. In the next section we propose a simple new method for solving the nonlinear system of equations.

\section{Successive linearisation method (SLM) solution}

The SLM algorithm starts with the assumption that the independent variables $f(\eta), \theta(\eta)$ and $\phi(\eta)$ can be expressed as

$$
f(\eta)=f_{i}(\eta)+\sum_{n=0}^{i-1} f_{n}(\eta), \quad \theta(\eta)=\theta_{i}(\eta)+\sum_{n=0}^{i-1} \theta_{n}(\eta), \phi(\eta)=\phi_{i}(\eta)+\sum_{n=0}^{i-1} \phi_{n}(\eta)
$$

where $f_{i}, \theta_{i}, \phi_{i}(i=1,2,3, \ldots)$ are unknown functions and $f_{n}, \theta_{n}$ and $\phi_{n}(n \geq 1)$ are approximations which are obtained by recursively solving the linear part of the equation system that results from substituting (15) in the governing equations. The main assumption of the SLM is that $f_{i}, \theta_{i}$ and $\phi_{i}$ become increasingly smaller when $i$ becomes large. that is

$$
\lim _{i \rightarrow \infty} f_{i}=\lim _{i \rightarrow \infty} \theta_{i}=\lim _{i \rightarrow \infty} \phi_{i}=0 .
$$

Thus, starting from the initial guesses $f_{0}(\eta), \phi_{0}(\eta), \theta_{0}(\eta)$, which are chosen to satisfy boundary conditions, the subsequent solutions for $f_{i}, \phi_{i}, \theta_{i} i \geq 1$ are obtained by successively solving the linearised form of the equations which are obtained by substituting equation (15) in the governing equations and considering only the linear terms. The linearised equations to be solved are given as

$$
\begin{aligned}
a_{1, i-1} f_{i}^{\prime \prime}+a_{2, i-1} f_{i}^{\prime}-\theta_{i}^{\prime}-N_{1} \phi_{i}^{\prime} & =r_{1, i-1}, \\
\theta_{i}^{\prime \prime}+b_{1, i-1} \theta_{i}^{\prime}+b_{2, i-1} f_{i}+D_{f} \phi_{i}^{\prime \prime} & =r_{2, i-1}, \\
\phi_{i}^{\prime \prime}+c_{1, i-1} \phi_{i}^{\prime}+c_{2, i-1} f_{i}+\operatorname{LeSr} \theta_{i}^{\prime \prime} & =r_{3, i-1},
\end{aligned}
$$

subject to the boundary conditions

$$
f_{i}(0)=f_{i}^{\prime}(\infty)=0, \theta_{i}(0)=\theta_{i}(\infty)=0, \phi_{i}(0)=\phi_{i}(\infty)=0 .
$$

where the coefficient parameters $a_{k, i-1}, b_{k, i-1}, c_{k, i-1}(k=1,2), r_{j, i-1}(j=1,2,3)$ are defined as, 


$$
\begin{aligned}
a_{1, i-1}= & 1+2 G r^{*} \sum_{n=0}^{i-1} f_{n}^{\prime}, \quad a_{2, i-1}=2 G r^{*} \sum_{n=0}^{i-1} f_{n}^{\prime \prime}, \\
b_{1, i-1}= & \frac{1}{2} \sum_{n=0}^{i-1} f_{n}, \quad b_{2, i-1}=\frac{1}{2} \sum_{n=0}^{i-1} \theta_{n}^{\prime}, \\
c_{1, i-1}= & \frac{1}{2} \sum_{n=0}^{i-1} f_{n}, \quad c_{2, i-1}=\frac{L e}{2} \sum_{n=0}^{i-1} \phi_{n}^{\prime}, \\
r_{1, i-1}= & \sum_{n=0}^{i-1} \theta_{n}^{\prime}+N_{1} \sum_{n=0}^{i-1} \phi_{n}^{\prime}-\sum_{n=0}^{i-1} f_{n}^{\prime \prime}-2 G r^{*} \sum_{n=0}^{i-1} f_{n}^{\prime} \sum_{n=0}^{i-1} f_{n}^{\prime \prime}, \\
r_{2, i-1}= & -\sum_{n=0}^{i-1} \theta_{n}^{\prime \prime}-\frac{1}{2} \sum_{n=0}^{i-1} f_{n} \sum_{n=0}^{i-1} \theta_{n}^{\prime}-D_{f} \sum_{n=0}^{i-1} \phi_{n}^{\prime \prime}, \\
r_{3, i-1}= & -\sum_{n=0}^{i-1} \phi_{n}^{\prime \prime}-\frac{L e}{2} \sum_{n=0}^{i-1} f_{n} \sum_{n=0}^{i-1} \phi_{n}^{\prime}-L e S_{r} \sum_{n=0}^{i-1} \theta_{n}^{\prime \prime} .
\end{aligned}
$$

Once each solution for $f_{i}, \theta_{i}, \phi_{i}(i \geq 1)$ has been found from iteratively solving equations (17 20), the approximate solutions for $f(\eta), \theta(\eta)$ and $\phi(\eta)$ are obtained as

$$
f(\eta) \approx \sum_{m=0}^{M} f_{m}(\eta), \quad \theta(\eta) \approx \sum_{m=0}^{M} \theta_{m}(\eta), \phi(\eta) \approx \sum_{m=0}^{M} \phi_{m}(\eta),
$$

where $M$ is the order of SLM approximation. Since the coefficient parameters and the right hand side of equations (17 - 19), for $i=1,2,3, \ldots$, are known (from previous iterations). The equation system can easily be solved analytically (wherever possible) or using any numerical method such as finite differences, finite elements, Runge-Kutta based shooting methods or collocation methods. In this work, equations (17 - 20) are solved using the Chebyshev spectral collocation method. This method is based on approximating the unknown functions by the Chebyshev interpolating polynomials in such a way that they are collocated at the Gauss-Lobatto points defined as

$$
\xi_{j}=\cos \frac{\pi j}{N}, \quad j=0,1, \ldots, N .
$$

where $N+1$ is the number of collocation points used (see for example Canuto et al. (1988); Don \& Solomonoff (1995); Trefethen (2000)). In order to implement the method, the physical region $[0, \infty)$ is transformed into the region $[-1,1]$ using the domain truncation technique in which the problem is solved on the interval $[0, L]$ instead of $[0, \infty)$. This leads to the mapping

$$
\frac{\eta}{L}=\frac{\xi+1}{2}, \quad-1 \leq \xi \leq 1
$$

where $L$ is the scaling parameter used to invoke the boundary condition at infinity. The unknown functions $f_{i}, \theta_{i}$ and $\phi_{i}$ are approximated at the collocation points by

$$
f_{i}(\xi) \approx \sum_{k=0}^{N} f_{i}\left(\xi_{k}\right) T_{k}\left(\xi_{j}\right), \theta_{i}(\xi) \approx \sum_{k=0}^{N} \theta_{i}\left(\xi_{k}\right) T_{k}\left(\xi_{j}\right), \phi_{i}(\xi) \approx \sum_{k=0}^{N} \phi_{i}\left(\xi_{k}\right) T_{k}\left(\xi_{j}\right), j=0,1, \ldots, N
$$


where $T_{k}$ is the $k$ th Chebyshev polynomial defined as

$$
T_{k}(\xi)=\cos \left[k \cos ^{-1}(\xi)\right] .
$$

The derivatives of the variables at the collocation points are represented as

$$
\frac{d^{a} f_{i}}{d \eta^{a}}=\sum_{k=0}^{N} \mathbf{D}_{k j}^{a} f_{i}\left(\xi_{k}\right), \quad \frac{d^{a} \theta_{i}}{d \eta^{a}}=\sum_{k=0}^{N} \mathbf{D}_{k j}^{a} \theta_{i}\left(\xi_{k}\right), \frac{d^{a} \phi_{i}}{d \eta^{a}}=\sum_{k=0}^{N} \mathbf{D}_{k j}^{a} \phi_{i}\left(\xi_{k}\right), j=0,1, \ldots, N
$$

where $a$ is the order of differentiation and $\mathbf{D}=\frac{2}{L} \mathcal{D}$ with $\mathcal{D}$ being the Chebyshev spectral differentiation matrix (see for example, Canuto et al. (1988); Trefethen (2000)). Substituting equations $(29$ - 32) in (17) - (20) leads to the matrix equation given as

$$
\mathbf{A}_{i-1} \mathbf{X}_{i}=\mathbf{R}_{i-1}
$$

subject to the boundary conditions

$$
f_{i}\left(\xi_{N}\right)=\sum_{k=0}^{N} \mathbf{D}_{0 k} f_{i}\left(\xi_{k}\right)=\theta_{i}\left(\xi_{N}\right)=\theta_{i}\left(\xi_{0}\right)=\phi_{i}\left(\xi_{N}\right)=\phi_{i}\left(\xi_{0}\right)=0
$$

in which $\mathbf{A}_{i-1}$ is a $(3 N+3) \times(3 N+3)$ square matrix and $\mathbf{X}_{i}$ and $\mathbf{R}_{i-1}$ are $(3 N+1) \times 1$ column vectors defined by

$$
\mathbf{A}_{i-1}=\left[\begin{array}{ccc}
A_{11} & A_{12} & A_{13} \\
A_{21} & A_{22} & A_{23} \\
A_{31} & A_{32} & A_{33}
\end{array}\right], \quad \mathbf{X}_{i}=\left[\begin{array}{c}
\mathbf{F}_{i} \\
\mathbf{\Theta}_{i} \\
\boldsymbol{\Phi}_{i}
\end{array}\right], \quad \mathbf{R}_{i-1}=\left[\begin{array}{c}
\mathbf{r}_{1, i-1} \\
\mathbf{r}_{2, i-1} \\
\mathbf{r}_{3, i-1}
\end{array}\right],
$$

where

$$
\begin{aligned}
\mathbf{F}_{i} & =\left[f_{i}\left(\xi_{0}\right), f_{i}\left(\xi_{1}\right), \ldots, f_{i}\left(\xi_{N-1}\right), f_{i}\left(\xi_{N}\right)\right]^{T}, \\
\boldsymbol{\Theta}_{i} & =\left[\theta_{i}\left(\xi_{0}\right), \theta_{i}\left(\xi_{1}\right), \ldots, \theta_{i}\left(\xi_{N-1}\right), \theta_{i}\left(\xi_{N}\right)\right]^{T}, \\
\mathbf{\Phi}_{i} & =\left[\phi_{i}\left(\xi_{0}\right), \phi_{i}\left(\xi_{1}\right), \ldots, \phi_{i}\left(\xi_{N-1}\right), \phi_{i}\left(\xi_{N}\right)\right]^{T}, \\
\mathbf{r}_{1, i-1} & =\left[r_{1, i-1}\left(\xi_{0}\right), r_{1, i-1}\left(\xi_{1}\right), \ldots, r_{1, i-1}\left(\xi_{N-1}\right), r_{1, i-1}\left(\xi_{N}\right)\right]^{T}, \\
\mathbf{r}_{2, i-1} & =\left[r_{2, i-1}\left(\xi_{0}\right), r_{2, i-1}\left(\xi_{1}\right), \ldots, r_{2, i-1}\left(\xi_{N-1}\right), r_{2, i-1}\left(\xi_{N}\right)\right]^{T}, \\
\mathbf{r}_{3, i-1} & =\left[r_{3, i-1}\left(\xi_{0}\right), r_{3, i-1}\left(\xi_{1}\right), \ldots, r_{3, i-1}\left(\xi_{N-1}\right), r_{3, i-1}\left(\xi_{N}\right)\right]^{T}, \\
A_{11} & =\mathbf{a}_{1, i-1} \mathbf{D}^{2}+\mathbf{a}_{2, i-1} \mathbf{D}, A_{12}=-\mathbf{D}, A_{13}=-N_{1} \mathbf{D} \\
A_{21} & =\mathbf{b}_{2, i-1}, A_{22}=\mathbf{D}^{2}+\mathbf{b}_{1, i-1} \mathbf{D}, \quad A_{23}=D_{f} \mathbf{D}^{2}, \\
A_{31} & =\mathbf{c}_{2, i-1}, \quad A_{32}=L_{e} S_{r} \mathbf{D}^{2}, \quad A_{33}=\mathbf{D}^{2}+\mathbf{c}_{1, i-1} \mathbf{D} .
\end{aligned}
$$

In the above definitions, $\mathbf{a}_{k, i-1}, \mathbf{b}_{k, i-1}, \mathbf{c}_{k, i-1}(k=1,2)$ are diagonal matrices of size $(N+1) \times$ $(N+1)$ and the superscript $T$ is the transpose.

The boundary conditions (34) are imposed on equation (33) by modifying the first and last rows of $\mathbf{A}_{m n}(m, n=1,2,3)$ and $\mathbf{r}_{m, i-1}$ in such a way that the modified matrices $\mathbf{A}_{i-1}$ and $\mathbf{R}_{i-1}$ take the form; 


\begin{tabular}{|c|c|c|c|c|c|c|c|c|c|c|c|c|c|c|c|}
\hline & $\mathbf{D}_{0,0}$ & $\mathbf{D}_{0,1}$ & $\begin{array}{l}\cdots \\
\mathbf{A}_{1}\end{array}$ & $\mathbf{D}_{0, N-1}$ & $\mathbf{D}_{0, N}$ & 0 & 0 & $\begin{array}{l}\cdots \\
\mathbf{A}_{12}\end{array}$ & 0 & 0 & 0 & 0 & $\begin{array}{c}\cdots \\
\mathbf{A}_{13}\end{array}$ & 0 & 0 \\
\hline & 0 & 0 & $\ldots$ & 0 & 1 & 0 & 0 & $\ldots$ & 0 & 0 & 0 & 0 & $\ldots$ & 0 & 0 \\
\hline & 0 & 0 & $\cdots$ & 0 & 0 & 1 & 0 & $\cdots$ & 0 & 0 & 0 & 0 & $\cdots$ & 0 & $\overline{0}$ \\
\hline $\mathbf{A}_{i-1}=$ & & & $\mathbf{A}_{2}$ & & & & & $A_{22}$ & & & & & $A_{23}$ & & \\
\hline & 0 & 0 & $\ldots$ & 0 & 0 & 0 & 0 & $\ldots$ & 0 & 1 & 0 & 0 & $\ldots$ & 0 & 0 \\
\hline & 0 & 0 & $\ldots$ & 0 & 0 & 0 & 0 & $\cdots$ & 0 & 0 & 1 & 0 & $\ldots$ & 0 & 0 \\
\hline & & & $\mathbf{A}_{3}$ & & & & & & & & & & & & \\
\hline & 0 & 0 & $\ldots$ & 0 & 0 & 0 & 0 & $\ldots$ & 0 & 0 & 0 & 0 & $\ldots$ & 0 & \\
\hline
\end{tabular}

$$
\mathbf{R}_{i-1}=\left(\begin{array}{c}
0 \\
r_{1, i-1}\left(\xi_{1}\right) \\
\vdots \\
r_{1, i-1}\left(\xi_{N-2}\right) \\
r_{1, i-1}\left(\xi_{N-1}\right) \\
0 \\
\hline 0 \\
r_{2, i-1}\left(\xi_{1}\right) \\
\vdots \\
r_{2, i-1}\left(\xi_{N-2}\right) \\
r_{2, i-1}\left(\xi_{N-1}\right) \\
0 \\
\hline 0 \\
r_{3, i-1}\left(\xi_{1}\right) \\
\vdots \\
r_{3, i-1}\left(\xi_{N-2}\right) \\
r_{3, i-1}\left(\xi_{N-1}\right) \\
0
\end{array}\right)
$$

After modifying the matrix system (33) to incorporate boundary conditions, the solution is obtained as

$$
\mathbf{X}_{i}=\mathbf{A}_{i-1}^{-1} \mathbf{R}_{i-1}
$$




\section{Results and discussion}

In this section we give the successive linearization method results for the main parameters affecting the flow. To check the accuracy of the proposed successive linearisation method (SLM), comparison was made with numerical solutions obtained using the MATLAB routine bvp4c, which is an adaptive Lobatto quadrature scheme. The graphs and tables presented in this work, unless otherwise specified, were generated using $N=150, L=30, N_{1}=1, L e=1$, $D_{f}=1, G r=1$ and $S_{r}=0.5$

Tables 1 - 8 give results for the Nusselt and Sherwood numbers at different orders of approximation when varying the values of the main parameters. Table 1 and 2 depict the numerical values of the local Nusselt Number and the Sherwood number, respectively, for various modified Grashof numbers. In this chapter, the modified Grashof numbers are used to evaluate the relative importance of inertial effects and viscous effects. It is clearly observed that the local Nusselt number and the local Sherwood number tend to decrease as the modified Grashof number $\mathrm{Gr}^{*}$ increases. Increasing $\mathrm{Gr}^{*}$ values retards the flow, thereby thickening the thermal and concentration boundary layers and thus reducing the heat and mass transfer rates between the fluid and the wall. We also observe in both of these tables that the successive linearisation method rapidly converges to a fixed value.

\begin{tabular}{ccccccc}
\hline$G r^{*}$ & 2nd order & 3rd order & 4th order & 6th order & 8th order & 10th order \\
\hline 0.5 & 0.25449779 & 0.25459016 & 0.25459014 & 0.25459014 & 0.25459014 & 0.25459014 \\
1.0 & 0.23356479 & 0.23357092 & 0.23357092 & 0.23357092 & 0.23357092 & 0.23357092 \\
1.5 & 0.21998679 & 0.21998820 & 0.21998820 & 0.21998820 & 0.21998820 & 0.21998820 \\
2.0 & 0.21001909 & 0.21001959 & 0.21001959 & 0.21001959 & 0.21001959 & 0.21001959 \\
2.5 & 0.20218859 & 0.20218881 & 0.20218881 & 0.20218881 & 0.20218881 & 0.20218881 \\
3.0 & 0.19576817 & 0.19576829 & 0.19576829 & 0.19576829 & 0.19576829 & 0.19576829 \\
\hline
\end{tabular}

Table 1. Values of the Nusselt Number, $-\theta^{\prime}(0)$ for different values of $G r^{*}$ at different orders of the SLM approximation using $L=30, N=150$ when $L e=1, N_{1}=1, D_{f}=1, S_{r}=0.5$

Table 3 and 4 represent the numerical values of the local Nusselt number and Sherwood number, respectively, for various buoyancy ratios $\left(N_{1}\right)$. We observe that the local Nusselt number and Sherwood number tend to increase as the buoyancy ratio $N_{1}$ increases. Increasing the buoyancy ratio accelerates the flow, decreasing the thermal and concentration boundary layer thickness and thus increasing the heat and mass transfer rates between the fluid and the wall.

\begin{tabular}{ccccccc}
\hline$G r^{*}$ & 2nd order & 3rd order & 4th order & 6th order & 8th order & 10th order \\
\hline 0.5 & 0.49979076 & 0.49970498 & 0.49970494 & 0.49970494 & 0.49970494 & 0.49970494 \\
1.0 & 0.45388857 & 0.45388333 & 0.45388333 & 0.45388333 & 0.45388333 & 0.45388333 \\
1.5 & 0.42518839 & 0.42518709 & 0.42518709 & 0.42518709 & 0.42518709 & 0.42518709 \\
2.0 & 0.40449034 & 0.40448985 & 0.40448985 & 0.40448985 & 0.40448985 & 0.40448985 \\
2.5 & 0.38841782 & 0.38841759 & 0.38841759 & 0.38841759 & 0.38841759 & 0.38841759 \\
3.0 & 0.37534971 & 0.37534959 & 0.37534959 & 0.37534959 & 0.37534959 & 0.37534959 \\
\hline
\end{tabular}

Table 2. Values of the Sherwood Number, $-\phi^{\prime}(0)$ for different values of $G r^{*}$ at different orders of the SLM approximation using $L=30, N=150$ when $L e=1, N_{1}=1, D_{f}=1, S_{r}=0.5$

Table 5 and 6 show the values of the local Nusselt number and local Sherwood number, respectively for various values of the Soret number $S r$. It is noticed that the magnitude of the local Nusselt number increases for $S r$ values less than a unit. However it decreases for 
Successive Linearisation Solution of

\begin{tabular}{ccccccc}
\hline$N_{1}$ & 2nd order & 3rd order & 4th order & 6th order & 8th order & 10th order \\
\hline 0 & 0.10955207 & 0.13941909 & 0.17140588 & 0.18806653 & 0.18822338 & 0.18822338 \\
1 & 0.23329934 & 0.23357050 & 0.23357092 & 0.23357092 & 0.23357092 & 0.23357092 \\
2 & 0.26550968 & 0.26554932 & 0.26554933 & 0.26554933 & 0.26554933 & 0.26554933 \\
3 & 0.29071828 & 0.29072940 & 0.29072940 & 0.29072940 & 0.29072940 & 0.29072940 \\
4 & 0.31170490 & 0.31170917 & 0.31170917 & 0.31170917 & 0.31170917 & 0.31170917 \\
5 & 0.32980517 & 0.32980715 & 0.32980715 & 0.32980715 & 0.32980715 & 0.32980715 \\
10 & 0.39608249 & 0.39638607 & 0.39638637 & 0.39638637 & 0.39638637 & 0.39638637 \\
\hline
\end{tabular}

Table 3. Values of the Nusselt Number, $-\theta^{\prime}(0)$ for different values of $N_{1}$ at different orders of the SLM approximation using $L=30, N=150$ when $G r^{*}=1, L e=1, D_{f}=1, S_{r}=0.5$

\begin{tabular}{ccccccc}
\hline$N_{1}$ & 2nd order & 3rd order & 4th order & 6th order & 8th order & 10th order \\
\hline 0 & 0.43723544 & 0.39927412 & 0.37520193 & 0.36285489 & 0.36273086 & 0.36273086 \\
1 & 0.45378321 & 0.45388312 & 0.45388333 & 0.45388333 & 0.45388333 & 0.45388333 \\
2 & 0.51638584 & 0.51639273 & 0.51639273 & 0.51639273 & 0.51639273 & 0.51639273 \\
3 & 0.56500365 & 0.56500444 & 0.56500444 & 0.56500444 & 0.56500444 & 0.56500444 \\
4 & 0.60522641 & 0.60522641 & 0.60522641 & 0.60522641 & 0.60522641 & 0.60522641 \\
5 & 0.63977204 & 0.63977193 & 0.63977193 & 0.63977193 & 0.63977193 & 0.63977193 \\
10 & 0.76607162 & 0.76609949 & 0.76609963 & 0.76609963 & 0.76609963 & 0.76609963 \\
\hline
\end{tabular}

Table 4. Values of the Sherwood number, $-\phi^{\prime}(0)$ for different values of $N_{1}$ at different orders of the SLM approximation using $L=30, N=150$ when $G r^{*}=1, L e=1, D_{f}=1, S_{r}=0.5$

larger values of $\mathrm{Sr}$. The magnitude of the local Sherwood number decreases for Soret number values less than unit but increases for large values of $S r$.

As the Dufour effect $D f$ increases (Table 7 and 8 ) heat transfer decreases and mass transfer increases.

Figure 1 illustrates the temperature and concentration profiles as a function of the similarity variable $\eta$ for various values of the modified Grashof number. It is observed from these figures that an increase in the modified Grashof number leads to increases in the temperature and the concentration distributions in the boundary layer and as a result both the thermal and solutal boundary layers become thicker.

The effect of the Lewis number Le on the temperature and concentration distributions are shown in Figure 2. We observe here that the temperature increases with increases values of Le for small values of the similarity variable $\eta(<4)$. Thereafter, the temperature decreases with increasing values of $L e$. We observe further that the species concentration distributions

\begin{tabular}{ccccccc}
\hline$S_{r}$ & 2nd order & 3rd order & 4th order & 6th order & 8th order & 10th order \\
\hline 0.0 & 0.11828553 & 0.15103149 & 0.18809470 & 0.20356483 & 0.20360093 & 0.20360093 \\
0.5 & 0.23358338 & 0.23357092 & 0.23357092 & 0.23357092 & 0.23357092 & 0.23357092 \\
1.5 & 0.28601208 & 0.28601478 & 0.28601478 & 0.28601478 & 0.28601478 & 0.28601478 \\
2.0 & 0.25626918 & 0.25626899 & 0.25626899 & 0.25626899 & 0.25626899 & 0.25626899 \\
3.0 & 0.21828033 & 0.21825939 & 0.21825951 & 0.21825951 & 0.21825951 & 0.21825951 \\
4.0 & 0.19258954 & 0.19260872 & 0.19260881 & 0.19260881 & 0.19260881 & 0.19260881 \\
5.0 & 0.17369571 & 0.17369607 & 0.17369607 & 0.17369607 & 0.17369607 & 0.17369607 \\
\hline
\end{tabular}

Table 5. Values of the Nusselt Number, $-\theta^{\prime}(0)$ for different values of $S_{r}$ at different orders of the SLM approximation using $L=30, N=150$ when $G r^{*}=1, L e=1, D_{f}=1, N_{1}=1$ 


\begin{tabular}{ccccccc}
\hline$S_{r}$ & 2nd order & 3rd order & 4th order & 6th order & 8th order & 10th order \\
\hline 0.0 & 0.57981668 & 0.51916309 & 0.50219360 & 0.49634082 & 0.49632751 & 0.49632751 \\
0.5 & 0.45386831 & 0.45388333 & 0.45388333 & 0.45388333 & 0.45388333 & 0.45388333 \\
1.5 & 0.35029249 & 0.35029514 & 0.35029514 & 0.35029514 & 0.35029514 & 0.35029514 \\
2.0 & 0.36241935 & 0.36241908 & 0.36241908 & 0.36241908 & 0.36241908 & 0.36241908 \\
3.0 & 0.37807254 & 0.37803636 & 0.37803657 & 0.37803657 & 0.37803657 & 0.37803657 \\
4.0 & 0.38517909 & 0.38521742 & 0.38521761 & 0.38521761 & 0.38521761 & 0.38521761 \\
5.0 & 0.38839482 & 0.38839561 & 0.38839562 & 0.38839562 & 0.38839562 & 0.38839562 \\
\hline
\end{tabular}

Table 6. Values of the Sherwood Number, $-\phi^{\prime}(0)$ for different values of $S_{r}$ at different orders of the SLM approximation using $L=30, N=150$ when $G r^{*}=1, L e=1, D_{f}=1, N_{1}=1$

\begin{tabular}{ccccccc}
\hline$D_{f}$ & 2nd order & 3rd order & 4th order & 6th order & 8th order & 10th order \\
\hline 0.0 & 0.53407939 & 0.49152600 & 0.48562621 & 0.48464464 & 0.48464458 & 0.48464458 \\
0.5 & 0.38477915 & 0.38479579 & 0.38479579 & 0.38479579 & 0.38479579 & 0.38479579 \\
0.8 & 0.30341973 & 0.30342078 & 0.30342078 & 0.30342078 & 0.30342078 & 0.30342078 \\
1.2 & 0.14317488 & 0.14317766 & 0.14317766 & 0.14317766 & 0.14317766 & 0.14317766 \\
1.4 & 0.01721331 & 0.01721348 & 0.01721348 & 0.01721348 & 0.01721348 & 0.01721348 \\
1.8 & -0.60409800 & -0.60409008 & -0.60409008 & -0.60409008 & -0.60409008 & -0.60409008 \\
\hline
\end{tabular}

Table 7. Values of the Nusselt Number, $-\theta^{\prime}(0)$ for different values of $D_{f}$ at different orders of the SLM approximation using $L=30, N=150$ when $G r^{*}=1, L e=1, S r=0.5, N_{1}=1$

\begin{tabular}{ccccccc}
\hline$D_{f}$ & 2nd order & 3rd order & 4th order & 6th order & 8th order & 10th order \\
\hline 0.0 & 0.32610052 & 0.32342809 & 0.33553652 & 0.33829291 & 0.33829308 & 0.33829308 \\
0.5 & 0.38480751 & 0.38479579 & 0.38479579 & 0.38479579 & 0.38479579 & 0.38479579 \\
0.8 & 0.42201071 & 0.42200998 & 0.42200998 & 0.42200998 & 0.42200998 & 0.42200998 \\
1.2 & 0.49527611 & 0.49527429 & 0.49527429 & 0.49527429 & 0.49527429 & 0.49527429 \\
1.4 & 0.55343701 & 0.55343690 & 0.55343690 & 0.55343690 & 0.55343690 & 0.55343690 \\
1.8 & 0.84855160 & 0.84854722 & 0.84854722 & 0.84854722 & 0.84854722 & 0.84854722 \\
\hline
\end{tabular}

Table 8. Values of the Sherwood Number, $-\phi^{\prime}(0)$ for different values of $D_{f}$ at different orders of the SLM approximation using $L=30, N=150$ when $G r^{*}=1, L e=1, S_{r}=0.5, N_{1}=1$
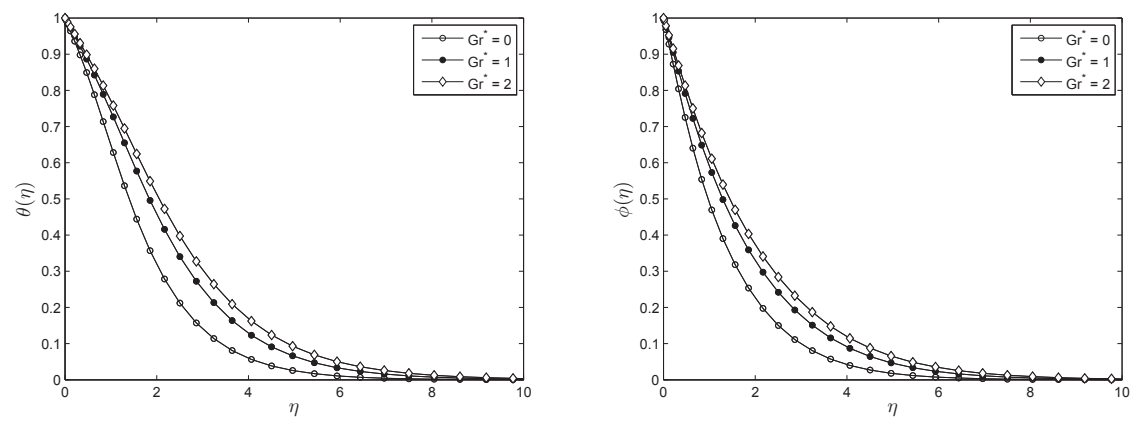

Fig. 1. Effect of $G r^{*}$ on the temperature and concentration profiles 

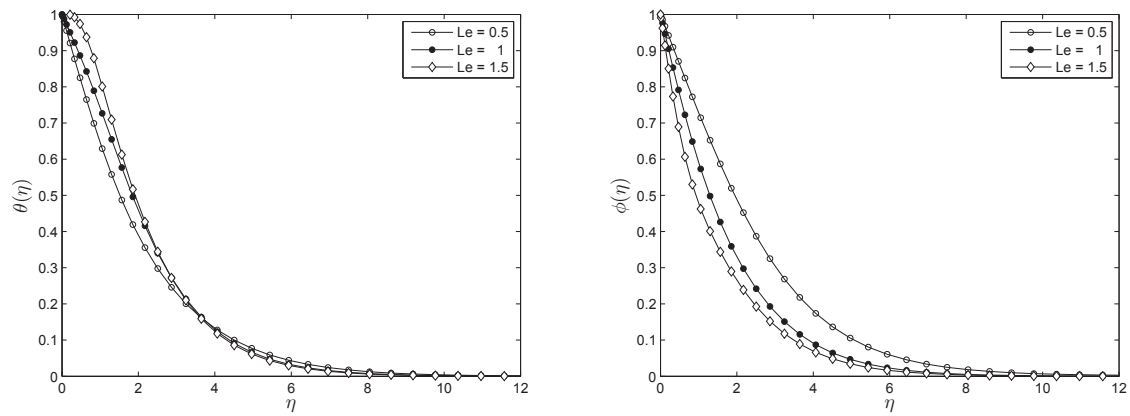

Fig. 2. Effect of Le on the temperature and concentration profiles
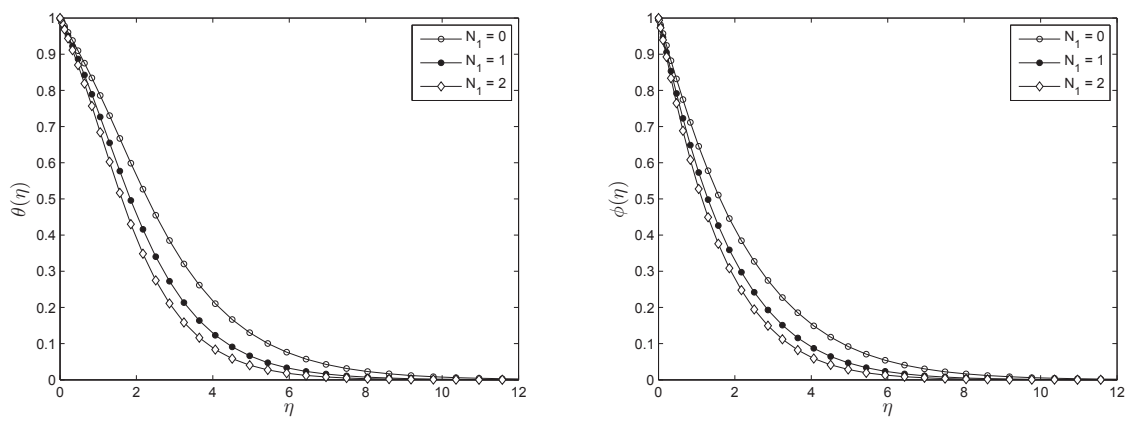

Fig. 3. Effect of $N_{1}$ on the temperature and concentration profiles

decrease due to an increase in the value of the Lewis number. Increasing Le leads to the thickening of the temperature boundary layer and to thin the concentration boundary layer. The temperature profiles and concentration profiles for aiding buoyancy are presented in Figure 3. It is seen in these figures that as the buoyancy parameter $N_{1}$ increases the temperature and concentration decrease. This is because the effect of the buoyancy ratio is to increase the surface heat and mass transfer rates. Therefore, the temperature and concentration gradients are increased and hence, so are the heat and mass transfer rates.

Figure 4 illustrates the effect of the Dufour parameter on the dimensionless temperature and concentration. It is observed that the temperature of fluid increases with an increase of Dufour number while the concentration of the fluid decreases with increases of the value of the Dufour number.

Figure 5 depict the effects of the Soret parameter on the dimensionless temperature and concentration distributions. It is clear from these figures that as the Soret parameters increases concentration profiles increase significantly while the temperature profiles decrease.

\section{Conclusion}

In the present chapter, a new numerical perturbation scheme for solving complex nonlinear boundary value problems arising in problems of heat and mass transfer. This numerical 

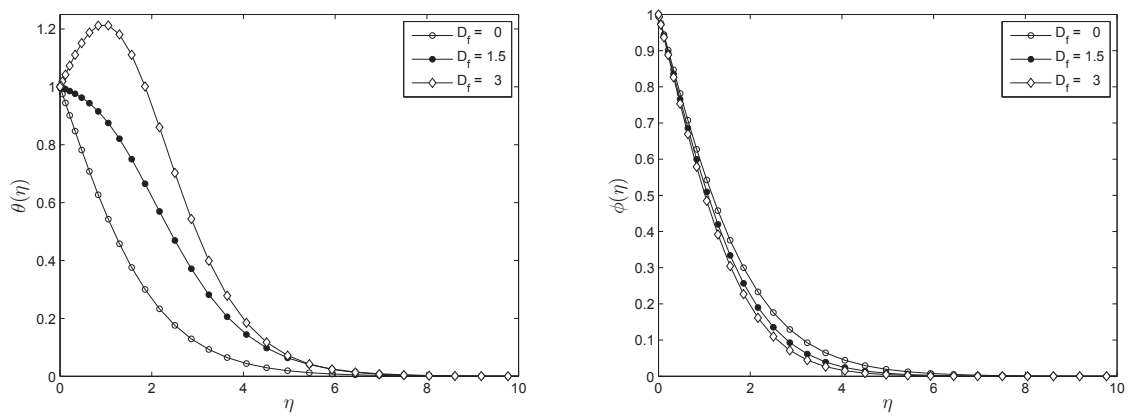

Fig. 4. Effect of $D_{f}$ on the temperature and concentration profiles
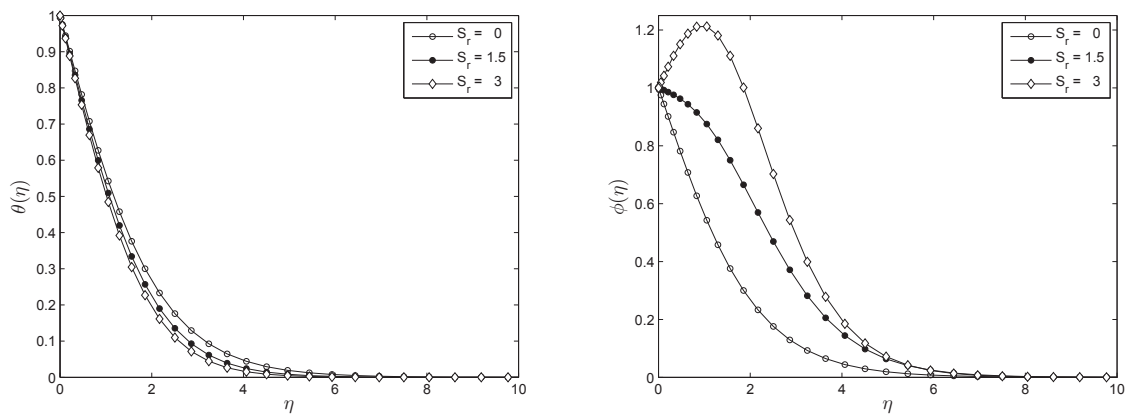

Fig. 5. Effect of $S_{r}$ on the temperature and concentration profiles

method is based on a novel idea of iteratively linearising the underlying governing non-linear boundary equations, which are written in similarity form, and then solving the resultant equations using spectral methods. Extensive numerical integrations were carried out, to investigate the non-Darcy natural convection heat and mass transfer from a vertical surface with heat and mass flux. The effects with the modified Grashof number, the buoyancy ratio, the Soret and Dufour numbers on the Sherwood and Nusselt numbers have been studied. From the present analysis, we conclude that (1) both the local Nusselt number, $N u_{x}$, and local Sherwood number, $S h_{x}$, decrease due to increase in the value of the inertial parameter (modified Grashof number, $G r^{*}$ ); (2) An increase in the buoyancy ratio tends to increase both the local Nusselt number and the Sherwood number; (3) The Lewis number has a more pronounced effect on the local mass transfer rate than it does on the local heat transfer rate; (4) Increases in Soret number tends to decrease the local heat transfer rate and the Dufour effects greatly affect the mass and heat transfer rates. Numerical results for the temperature and concentration were presented graphically. These results might find wide applications in engineering, such as geothermal system, heat exchangers, fibre and granular insulation, solar energy collectors and nuclear waste depositors. 


\section{References}

El-Amin, M.F. (2004) Double dispersion effects on natural convection heat and mass transfer in non-Darcy porous medium, Applied Mathematics and Computation Vol.156, 1-17

Adomian, G. (1976) Nonlinear stochastic differential equations. J Math. Anal. Appl. Vol.55, 441 $-52$

Adomian, G. (1991) A review of the decomposition method and some recent results for nonlinear equations. Comp. and Math. Appl. Vol.21, $101-27$

Ayaz,F. (2004) Solutions of the systems of differential equations by differential transform method, Applied Mathematics and Computation, Vol.147, 547-567

Canuto,C., Hussaini, M. Y., Quarteroni,A. and Zang, T. A. (1988) Spectral Methods in Fluid Dynamics, Springer-Verlag, Berlin

Chen,C.K., Ho,S.H. (1999) Solving partial differential equations by two dimensional differential transform method, Applied Mathematics and Computation Vol.106, 171-179.

Don, W. S., Solomonoff, A. (1995) Accuracy and speed in computing the Chebyshev Collocation Derivative. SIAM J. Sci. Comput, Vol.16, No.6, 1253-1268.

He,J.H, (1999) Homotopy perturbation technique, Comput. Methods Appl. Mech. Eng. Vol.178, $257-262$.

He,J.H. (2000) A new perturbation technique which is also valid for large parameters, J. Sound and Vibration, Vol.229, $1257-1263$.

He, J.H. (1999) Variational iteration method a kind of nonlinear analytical technique:some examples, Int. J. Nonlinear Mech. Vol.34, 699-708

He, J.H. (2006) New interpretation of homotopy perturbation method, Int. J. Modern Phys.B vol.20, $2561-2568$.

Liao,S.J. (1992) The proposed homotopy analysis technique for the solution of nonlinear problems, PhD thesis, Shanghai Jiao Tong University, 1992.

Liao,S.J. (1999) A uniformly valid analytic solution of 2D viscous flow past a semi-infinite flat plate. J. Fluid Mech. Vol.385, $101-128$.

Liao,S.J. (2003) Beyond perturbation: Introduction to homotopy analysis method. Chapman \& Hall/CRC Press.

Liao,S. J.(2009) Notes on the homotopy analysis method: Some definitions and theories, Commun. Nonlinear Sci. Numer. Simul. Vol.14, 983-997.

Motsa,S.S.,Sibanda, P., Shateyi,S. (2010) A new spectral-homotopy analysis method for solving a nonlinear second order BVP, Commun. Nonlinear Sci. Numer. Simul. Vol.15 2293-2302.

Motsa, S.S., Sibanda, P., Awad, F.G., Shateyi,S. (2010) A new spectral-homotopy analysis method for the MHD Jeffery-Hamel problem, Computer E Fluids Vol.39, 1219-1225.

Motsa,S.S., Shateyi,S., (2010) A New Approach for the Solution of Three-Dimensional Magnetohydrodynamic Rotating Flow over a Shrinking Sheet, Mathematical Problems in Engineering, vol. 2010, Article ID 586340, 15 pages, 2010. doi:10.1155/2010/586340

Makukula,Z., Sibanda,P., Motsa,S.S. (2010) A Note on the Solution of the Von Kármán Equations Using Series and Chebyshev Spectral Methods, Boundary Value Problems, Volume 2010 (2010), Article ID 471793, 17 pages doi:10.1155/2010/471793

Makukula,Z., Sibanda,P., Motsa,S.S. (2010) , A Novel Numerical Technique for Two-dimensional Laminar Flow Between Two Moving Porous Walls, Mathematical Problems in Engineering, Vol. 2010, Article ID 528956, 15 pages, 2010. doi: $10.1155 / 2010 / 528956$

Shateyi,S., Motsa,S.S.,(2010) Variable viscosity on magnetohydrodynamic fluid flow and heat 
transfer over an unsteady stretching surface with Hall effect, Boundary Value Problems, Vol. 2010, Article ID 257568, 20 pages, doi:10.1155/2010/257568

Murthy, P.V.S.N, Singh, P. (1999) Heat and mass transfer by natural convection in a non-Darcy porous medium, Acta Mechanica, Vol.138, 243-254

Lakhshmi Narayana, P.A., Murthy, P.V.S.N, (2006) Free convective heat and mass transfer in a doubly stratified non-Darcy porous medium, Journal of Heat Transfer, Vol.128, 1204-1212

Partha, M.K. (2008) Thermophoresis particle deposition in a non-Darcy porous medium under the influence of Soret, Dufour effects, Heat Mass Transfer Vol.44, 969-977

Trefethen, L.N. (2000) Spectral Methods in MATLAB, SIAM

Zhou, J.K. (1986) Differential Transformation and Its Applications for Electrical Circuits, Huazhong University Press, Wuhan, China (in Chinese) 


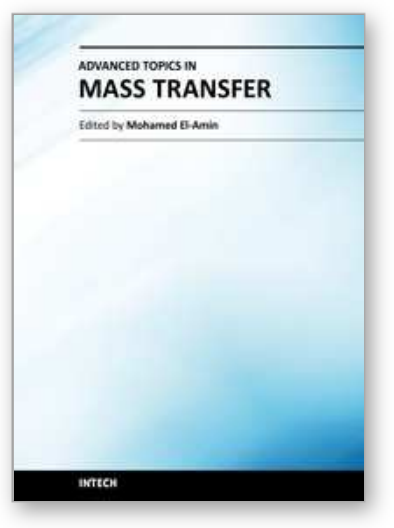

\author{
Advanced Topics in Mass Transfer \\ Edited by Prof. Mohamed El-Amin
}

ISBN 978-953-307-333-0

Hard cover, 626 pages

Publisher InTech

Published online 21, February, 2011

Published in print edition February, 2011

This book introduces a number of selected advanced topics in mass transfer phenomenon and covers its theoretical, numerical, modeling and experimental aspects. The 26 chapters of this book are divided into five parts. The first is devoted to the study of some problems of mass transfer in microchannels, turbulence, waves and plasma, while chapters regarding mass transfer with hydro-, magnetohydro- and electro- dynamics are collected in the second part. The third part deals with mass transfer in food, such as rice, cheese, fruits and vegetables, and the fourth focuses on mass transfer in some large-scale applications such as geomorphologic studies. The last part introduces several issues of combined heat and mass transfer phenomena. The book can be considered as a rich reference for researchers and engineers working in the field of mass transfer and its related topics.

\title{
How to reference
}

In order to correctly reference this scholarly work, feel free to copy and paste the following:

Sandile Motsa and Stanford Shateyi (2011). Successive Linearisation Solution of Free Convection Non-Darcy Flow with Heat and Mass Transfer, Advanced Topics in Mass Transfer, Prof. Mohamed El-Amin (Ed.), ISBN: 978-953-307-333-0, InTech, Available from: http://www.intechopen.com/books/advanced-topics-in-masstransfer/successive-linearisation-solution-of-free-convection-non-darcy-flow-with-heat-and-mass-transfer

\section{INTECH}

open science | open minds

\author{
InTech Europe \\ University Campus STeP Ri \\ Slavka Krautzeka 83/A \\ 51000 Rijeka, Croatia \\ Phone: +385 (51) 770447 \\ Fax: +385 (51) 686166 \\ www.intechopen.com
}

\author{
InTech China \\ Unit 405, Office Block, Hotel Equatorial Shanghai \\ No.65, Yan An Road (West), Shanghai, 200040, China \\ 中国上海市延安西路65号上海国际贵都大饭店办公楼 405 单元 \\ Phone: +86-21-62489820 \\ Fax: +86-21-62489821
}


(C) 2011 The Author(s). Licensee IntechOpen. This chapter is distributed under the terms of the Creative Commons Attribution-NonCommercialShareAlike-3.0 License, which permits use, distribution and reproduction for non-commercial purposes, provided the original is properly cited and derivative works building on this content are distributed under the same license. 